\title{
The genomic road to invasion-examining the similarities and differences in the genomes of associated oral pre-cancer and cancer samples
}

\author{
Henry M. Wood ${ }^{1 *}$, Catherine Daly ${ }^{1}$, Rebecca Chalkley ${ }^{1}$, Burcu Senguven ${ }^{4,1}$, Lisa Ross ${ }^{1}$, Philip Egan ${ }^{1,5}$, \\ Preetha Chengot ${ }^{2}$, Jennifer Graham², Neeraj Sethi ${ }^{1}$, Thian K. Ong ${ }^{3}$, Kenneth MacLennan ${ }^{1,2}$, Pamela Rabbitts ${ }^{1}$ \\ and Caroline Conway ${ }^{1,5}$
}

\begin{abstract}
Background: It is frequently assumed that pre-invasive lesions are simpler precursors of cancer and will contain a limited subset of the genomic changes seen in their associated invasive disease. Driver mutations are thought to occur early, but it is not known how many of these are present in pre-invasive lesions. These assumptions need to be tested with the increasing focus on both personalised cancer treatments and early detection methodologies.

Methods: We examined genomic copy number changes in 256 pre-invasive and invasive samples from 69 oral cancer patients. Forty-eight samples from 16 patients were further examined using exome sequencing.

Results: Evidence of a shared ancestor of both dysplasia and carcinoma was seen in all but one patient. One-third of dysplasias showed independent copy number events. The remainder had a copy number pattern that was similar to or simpler than that of the carcinoma. All dysplasias examined contained somatic mutations absent in the related carcinoma.

Previously observed copy number changes and TP53 mutations were very frequently observed, and almost always shared between dysplasia and carcinoma. Other gene changes were more sporadic. Pathway analysis confirmed that each patient's disease developed in a different way.

Examining the numbers of shared mutations and the rate of accumulation of mutations showed evidence that all samples contain a population of sub-clones, with little evidence of selective advantage of a subset of these.
\end{abstract}

Conclusions: These findings suggest that most of the genomic changes driving oral cancer occur in the pre-cancerous state by way of gradual random accumulation rather than a dramatic single event.

Keywords: Pre-cancer, Oral cancer, Tumour progression

\section{Background}

Malignant tumours have been observed to develop after passing through various pre-cancerous stages. Seminal work has suggested that pre-cancerous cells may need multiple mutations to become invasive, and that these may occur in a specific pattern, or one of a small number of patterns, depending on the cancer type $[1,2]$. Recent

\footnotetext{
* Correspondence: h.m.wood@leeds.ac.uk

'Leeds Institute of Cancer and Pathology, University of Leeds, Leeds LS9 7TF, UK

Full list of author information is available at the end of the article
}

advances in genomic technology have allowed more detailed analysis of the timing of these events in different cancer types $[3,4]$. Sophisticated mathematical models have been developed to infer the evolutionary dynamics of early and developing disease from fully invasive specimens $[5,6]$.

Pre-cancerous lesions are often difficult to study due to their small size and the feasibility of obtaining tissue. Head and neck squamous cell carcinoma (HNSCC), however, is an excellent disease in which to study pre-cancer, in that invasive carcinoma frequently presents alongside precancerous dysplasia, which is macroscopically visible and 
easily accessible due to its location. This has enabled several studies to examine groups of dysplasias and carcinomas [7-11]. Fortuitously, HNSCC may be one of the cancer subtypes that would benefit most from study of pre-cancer. The recent head and neck study of The Cancer Genome Atlas (TCGA) [12] showed that HNSCC has a particularly mixed set of genomic abnormalities, with few common driver genes. This suggests that the study of early disease instead may be a useful alternative approach.

One of the main subsets of HNSCC is oral cancer. Oral dysplasia is characterised by the presence of architectural and cytological atypia within the surface epithelium. Several classification and grading schemes are based on the World Health Organization (WHO) 2005 guidelines [13], each with varying predictive ability.

A novel binary system [14] for grading of oral dysplasia has the advantage of helping clinicians make treatment decisions for dysplastic lesions, especially those which would have been categorised as moderate dysplasia in the WHO 2005 classification.

Even though carcinoma is frequently described as arising from a bed of dysplasia, the relationship between dysplasias of various grades to each other and to their associated squamous cell carcinoma (SCC) is poorly understood. The genomic pathways from normal tissue through pre-cancerous stages to fully invasive disease has not been characterised in HNSCC. Work on small numbers of patients shows considerable variety in both the genomic evolutionary relationships and the differentially expressed pathways between HNSCC and their related dysplasias $[15,16]$.

In this study we attempted to better understand what genomic changes occur along the pathway to invasion, whether common patterns could be observed between patients and whether the view of gradually increasing grade leading to invasive disease would be reflected by genomic changes. We looked at gross genomic copy number changes in dysplasia and SCC samples from over 50 patients, and exome sequencing of a subset of those patients.

\section{Methods}

\section{Samples}

Patients with potential or proven cancerous oral lesions were recruited from the Oral Surgery outpatients' clinic at Leeds General Infirmary and were anonymised after providing informed consent. Blood samples were obtained at the time of diagnostic biopsy, and formalin-fixed paraffin-embedded (FFPE) blocks were subsequently obtained from the hospital archives.

Following sectioning, marking of areas of dysplasia and SCC (Additional file 1: Figure S1), and dissection, DNA was extracted using the Qiagen QIAamp DNA Mini Kit or QIAamp DNA micro kit. It was noted whether dysplasia samples were high (HGD) or low (LGD) grade, according to the binary grading system [14], and whether they were present in the same FFPE block as the SCC, or a different block, and therefore slightly further away, physically, from the SCC.

\section{Low coverage copy number sequencing}

Low coverage genomic sequence data were obtained for each patient using an updated version of previously published protocols [17]. DNA was prepared for Illumina sequencing using NEBNext multiplex library preparation kits incorporating custom designed tags. Forty libraries were pooled and sequenced together in one $2 \times 100 \mathrm{bp}$ Illumina HiSeq2500 lane, giving around $0.15 \times$ coverage per sample.

Sequencing reads were trimmed using cutadapt [18]. Genomic copy number data were produced by CNAnorm [19] after first aligning to the human reference genome (hg19) using BWA [20]. Breakpoints were called using DNAcopy [21]. Human papilloma virus (HPV) infection was tested by re-aligning the data to known viral genomes [22].

Tumour cell content was estimated using CNAnorm, in conjunction with histological examination. Adjusted copy number profiles were then compared as groups of similar samples (LGD, HGD, SCC) and between different samples from the same patient. The differences and/or similarities between dysplasias and associated SCCs were noted. The fraction of genome altered was calculated by summing the number of bases of autosomal genomic gain or loss and dividing by the size of the autosomal genome.

\section{Exome sequencing}

A subset of patients was selected for further analysis if their DNA from dysplasia and SCC was of sufficient quality for adequate copy number libraries to be produced, if the copy number data showed evidence of a disrupted genome, and if the samples proved to be HPV negative. All SCC samples had a tumour cell content of over $70 \%$. All dysplasia samples contained $100 \%$ dysplastic tissue.

For each patient, the exomes of matching blood, dysplasia and SCC were sequenced using the Sureselect Human all Exome Kit (Agilent) to an average of $125 \times$ coverage. Reads were trimmed using cutadapt, aligned to the human genome using BWA and processed using the GATK pipeline [23]. Mutations were called using Varscan2 [24] in somatic mode. Putative variants were initially filtered to remove any with a Varscan somatic score of 10 or below $(p>0.1)$ and any supported by fewer than three reads.

Following mutation calling, the variant allele frequency (VAF) of each mutation was calculated. The VAFs of 
mutations which were either unique to each sample or shared between dysplasia and SCC were compared both as histograms and dot plots. As mutation calls with a VAF $<0.12$ were more likely to be caused by sequencing errors or FFPE artefacts [6], these analyses were performed both with and without those calls with a VAF $<0.12$. The validity of this cutoff was confirmed by analysis of PCR validation of exome data from a similar cohort of previously published HNSCC FFPE samples [15] (Additional file 1: Figure S2).

\section{Examining neutral tumour evolution and mutation rate}

Following the recent work of Williams et al. [6], the VAFs of each sample were used to test for the presence or absence of neutral evolution. High VAF mutations $(>0.24)$ are more likely to be clonal so were removed, as were low values $(<0.12)$, which were more likely to be errors or artefacts. The remaining mutations represent the expanding and diversifying lesion and capture the subclonal architecture. The cumulative number of mutations, $M(f)$, was plotted against the inverse of the VAF, $1 / f$. The $R^{2}$ goodness of fit for each plot was calculated, with values over 0.98 indicating that the sample was following the neutral evolution model.

For those samples following the neutral model, mutation rates were calculated as the gradient of the $M(f)$ against $1 / \mathrm{f}$ plots divided by the $30-\mathrm{Mb}$ exome size, and expressed as mutations per base per effective cell division. This was calculated for all mutations and for each mutation type separately.

\section{Analysing functional variants}

To focus on those mutations which were likely to be driving the disease in each patient, mutations were filtered based on cellular frequency and possible effect on protein function The minimum possible cellular frequency of each mutation was calculated using the VAF, adjusted for normal cell contamination and copy number. For instance, in a triploid region of the genome in a sample with $70 \%$ tumour cell content, each tumour cell chromosomal copy will contribute $23.3 \%$ of the reads, so a mutation in over $50 \%$ of cells will have a minimum VAF of $11.7 \%$. The putative effects of each mutation were predicted using the Variant Effect Predictor [25]. Mutations were kept if they were present in over $50 \%$ of cells and had a somatic score of over $15(p<0.05)$, in either dysplasia or SCC, and if they had a possible effect on protein function, namely: anything affecting a splice site, frameshifts, stop gained or lost, coding deletions or insertions, initiator codon variants, incomplete terminal codon variants, exon elongations, truncation mutations, or missense mutations that were labelled as either possibly or probably damaging or deleterious. If a mutation passed filters in one sample but was present in lower cellular frequency in the paired sample, it was counted as present in both samples.

\section{Pathway analysis}

For each patient, lists of genes passing filters were split into three lists according to whether they were found in dysplasia only, SCC only or shared. These lists were compared to lists of genes from the $\operatorname{KEGG}[26,27]$ database in pathways previously associated with HNSCC: calcium signalling, cell cycle, DNA replication, ERBB signalling, JAK-STAT signalling, MAPK, NOTCH signalling, PI3K, P53 signalling, phosphatidylinositol signalling, VEGF signalling, WNT signalling and hedgehog. Lists of genes from HNSCC exome papers [28], the cancer gene census [29] and TCGA head and neck study [12] were also used.

A more agnostic approach was taken by importing the gene lists into the DAVID functional annotation tool $[30,31]$, and keeping those gene ontology (GO) terms that were enriched with a $p$ value of 0.05 or better.

\section{Results \\ Samples}

Data were collected from 69 patients. Fifty-nine patients provided enough DNA from both dysplasia and SCC samples for comparative analysis, totalling 256 samples (Additional file 1: Table S1).

\section{Copy number patterns}

The various LGD, HGD and SCC ( $n=38,59$ and 149, respectively) samples were examined individually and cumulatively. While there was considerable variation between samples, individually, LGD samples were more likely to have fewer genomic changes than HGD or SCC samples (median fractions genome altered were 0.12, 0.38 and 0.30 , respectively). A Mann-Whitney test comparing the distributions of fractions of genome altered between LGD and HGD samples gave a $p$ value of $4.76 \times 10^{-9}$. Comparing LGD and SCC samples gave a $p$ value of $2.57 \times 10^{-7}$, while comparing HGD and SCC only gave a $p$ value of 0.057 . This was confirmed by cumulative frequency plots (Fig. 1). The HGD and SCC groups were almost indistinguishable and matched the expected frequencies previously observed in TCGA HNSCC series. The mean copy number profiles of the two groups had a Pearson's correlation coefficient of 0.92. The correlations between the mean LGD copy number profile and the mean HGD and SCC profiles (both 0.81 ) were lower than the HGD/SCC comparison. Every region of common gain or loss in one group was reflected in the others. Only the relative frequencies of some of them varied slightly. When the respective frequencies of the most common regions of change in the three sample groups were compared (Table 1) most 


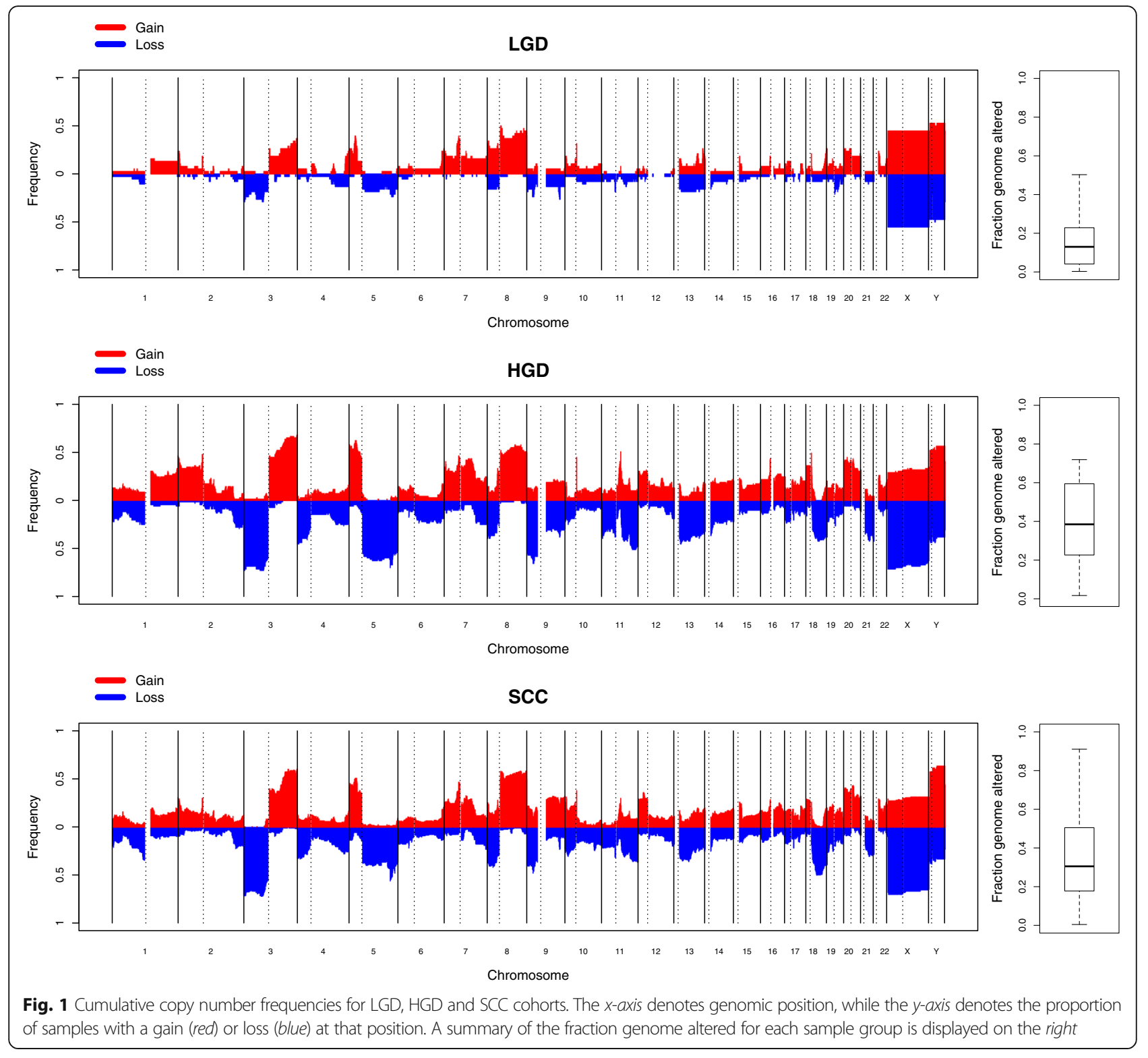

regions followed the expected pattern, predicted by the overall fraction of genome altered and sample numbers. Gain of chromosome 7 occurred in more LGD samples than would be expected, but this was not significant after adjusting for multiple testing. Only gain of chromosome arm 8q showed a significant deviation, again being present in more LGD samples than expected, indicating that, where present, it is a particularly early event.

Comparing patterns to those seen in TCGA data [12], these patients showed similar regions of gain and loss, albeit with a few differences. In the HPV-associated patients, neither amplification of the $E 2 F 1$ gene nor loss of TRAF were observed. In the HPV-negative patients, loss of TP53 was not observed. However, local gain of 3q26-
$3 \mathrm{q} 28$ was common, occurring in 23 out of 59 patients as either a partial arm gain or local amplification. Similarly, local amplification of 11q23 was observed in 23 patients.

Samples were also examined in the context of other samples from the same patient. For those patients from which LGD and HGD samples were analysed, the mean change across the genome seen in the HGD but not the matching LGD was calculated. A similar analysis was performed with patients with matching SCC and HGD samples (Fig. 2). For the LGD-HGD pairs, the changes seen in HGD samples but not their matched LGD samples very closely mirrored the cumulative frequency plot of SCC samples (correlation coefficient 0.82 ). In contrast, the changes seen in SCC samples but not the matched HGD 
Table 1 Percentage frequencies of the most common copy number events in LGD, HGD and SCC samples

\begin{tabular}{lllll}
\hline & LGD & HGD & SCC & $P$ value \\
\hline 3p loss & 28.95 & 72.46 & 0.72 & 0.3994 \\
4p loss & 13.16 & 44.93 & 0.33 & 0.9453 \\
5q loss & 23.68 & 69.57 & 0.56 & 0.9787 \\
9p loss & 26.32 & 65.22 & 0.50 & 0.7044 \\
11 pq loss & 18.42 & 50.72 & 0.36 & 0.7893 \\
18q loss & 7.89 & 42.03 & 0.50 & 0.0646 \\
3q gain & 36.84 & 68.12 & 0.60 & 0.2041 \\
5p gain & 39.47 & 62.32 & 0.51 & 0.0428 \\
7 pq gain & 39.47 & 47.83 & 0.50 & 0.0067 \\
8q gain & 50.00 & 57.97 & 0.59 & 0.0009 \\
12p gain & 7.89 & 31.88 & 0.36 & 0.2404 \\
Expected & 12 & 38 & 30 & \\
\hline
\end{tabular}

The $p$ values that each event differs from the expected frequencies were calculated from the overall fraction of genome altered and total sample number for each group using a chi-squared test. As 11 regions were tested, $p$ values below 0.0045 are considered significant samples appeared to be more random, with only a 0.51 correlation coefficient with the overall SCC pattern.

Patients were subdivided into groups according to the relationships between patterns of the dysplasia and SCC samples: (1) dysplasia and SCC showed some common features, but each displayed other independent features; (2) dysplasia and SCC were nearly identical; (3) the dysplasia showed a subset of the alterations seen in the SCC, or the dysplasia had a flat profile; (4) both dysplasia and SCC had flat profiles. Examples of these relationships are shown in Figs. 3, 4 and 5. Some patients provided more than one sample and could sometimes be classed into more than one of these groups, depending on which samples were compared.

Percentages of patients in each of these groups are shown in Fig. 6, as well as a patient-by-patient breakdown. Samples are classed according to whether the dysplasias were LGD or HGD and, separately, whether the dysplasias were in the same or a different block to the SCC. Some patients were counted multiple times. For example a patient might have had an adjacent HGD with pattern 1, an adjacent pattern 1 LGD and a distant pattern 2 LGD. This patient would be counted as pattern 1 in the HGD analysis, both 1 and 2 in the LGD

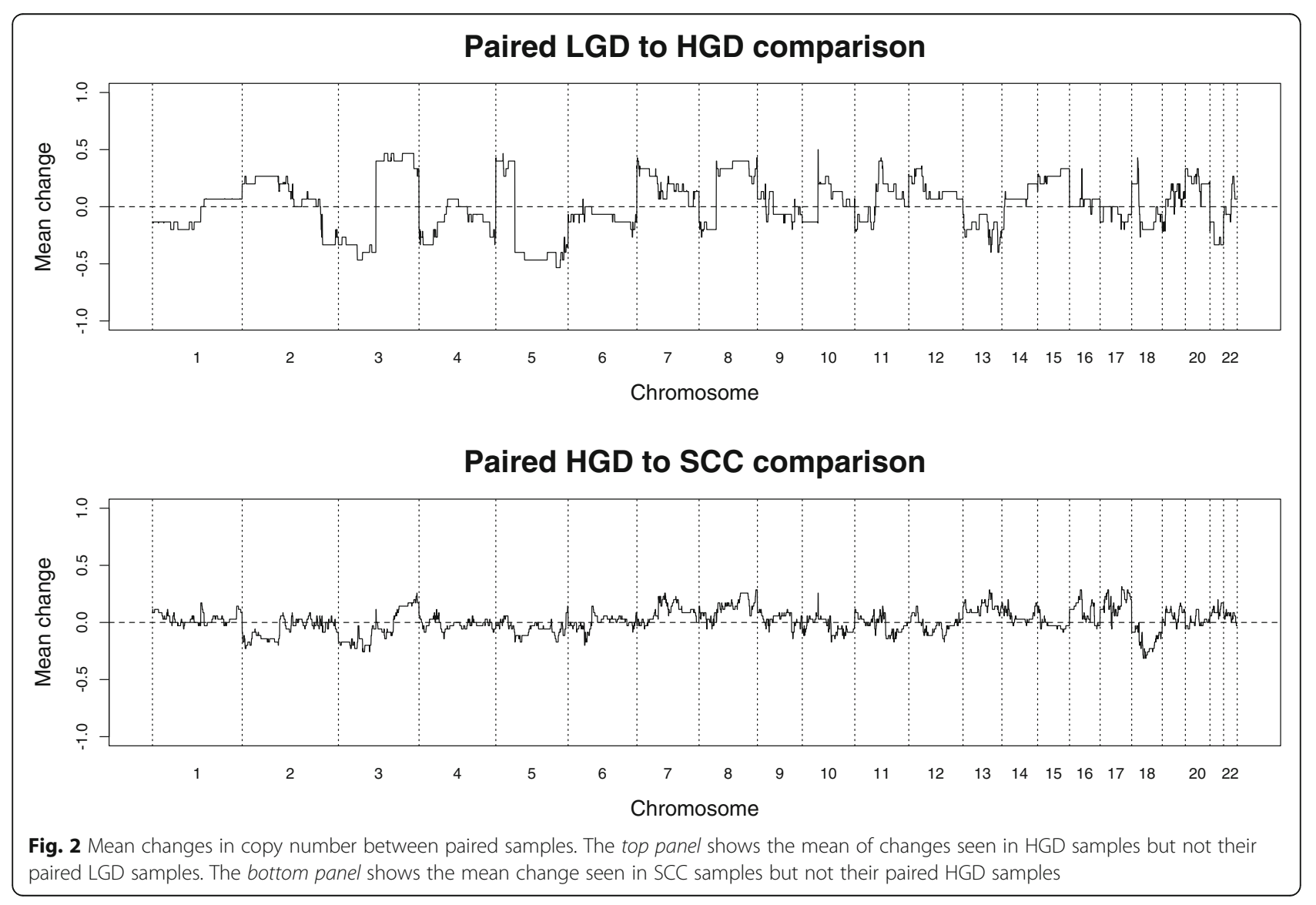




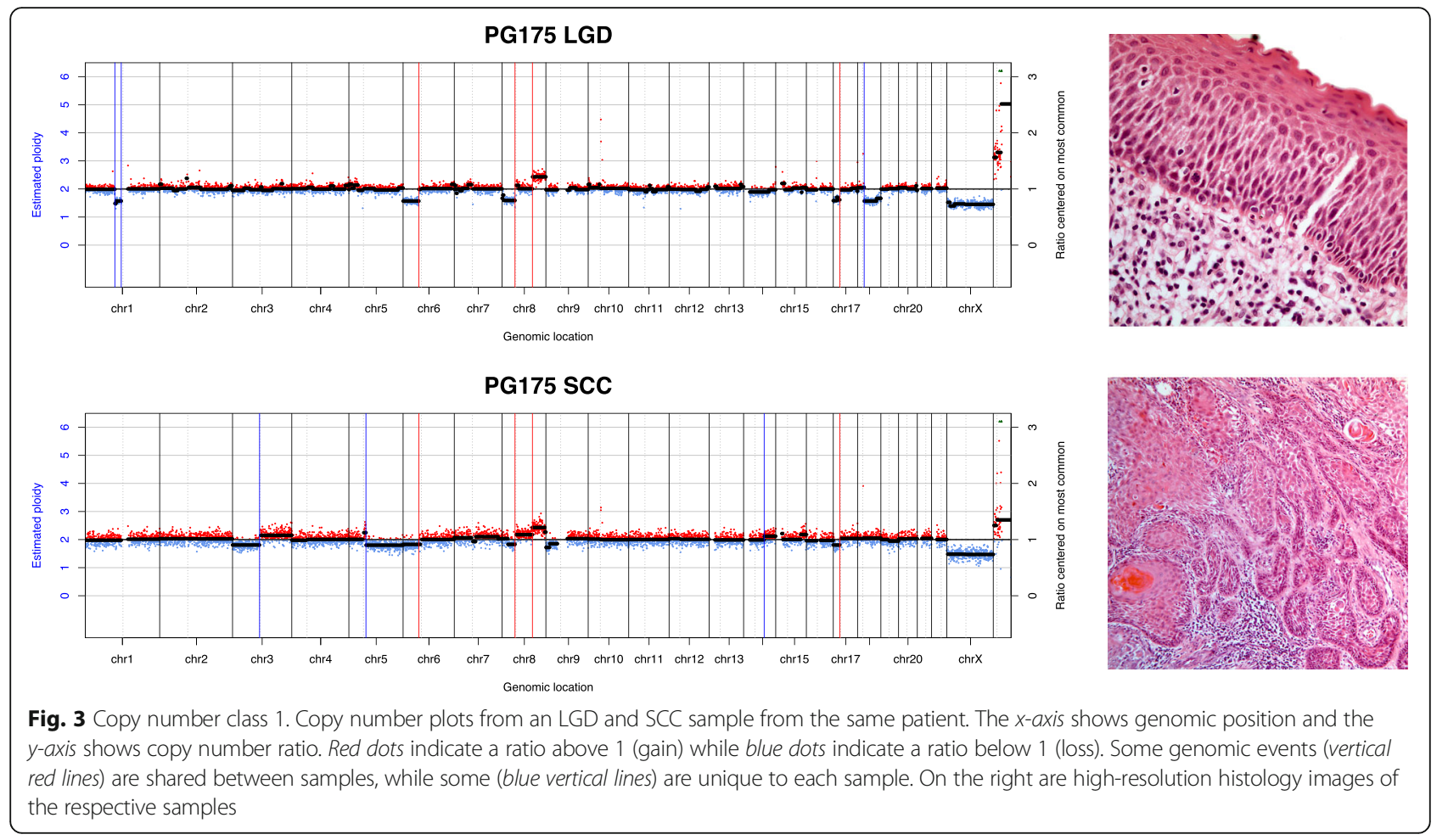

analysis, 1 for the adjacent dysplasia analysis and 2 for the distant dysplasia analysis.

Patterns 1, 2 and 3 accounted for all but one patient and were found at very similar overall frequencies. Comparing LGD and HGD samples, both types had very similar frequencies of pattern 1 (related but independent), the LGD samples had relatively higher frequencies of pattern 3 (dysplasia being a simpler version of the SCC), while the HGD samples had a relatively higher proportion of pattern 2 (SCC and dysplasia very similar). A chi-squared test showed this result to be significant $(p=0.0264)$.

When comparing samples with distant and adjacent dysplasia-SCC pairs, the distant samples had a relatively

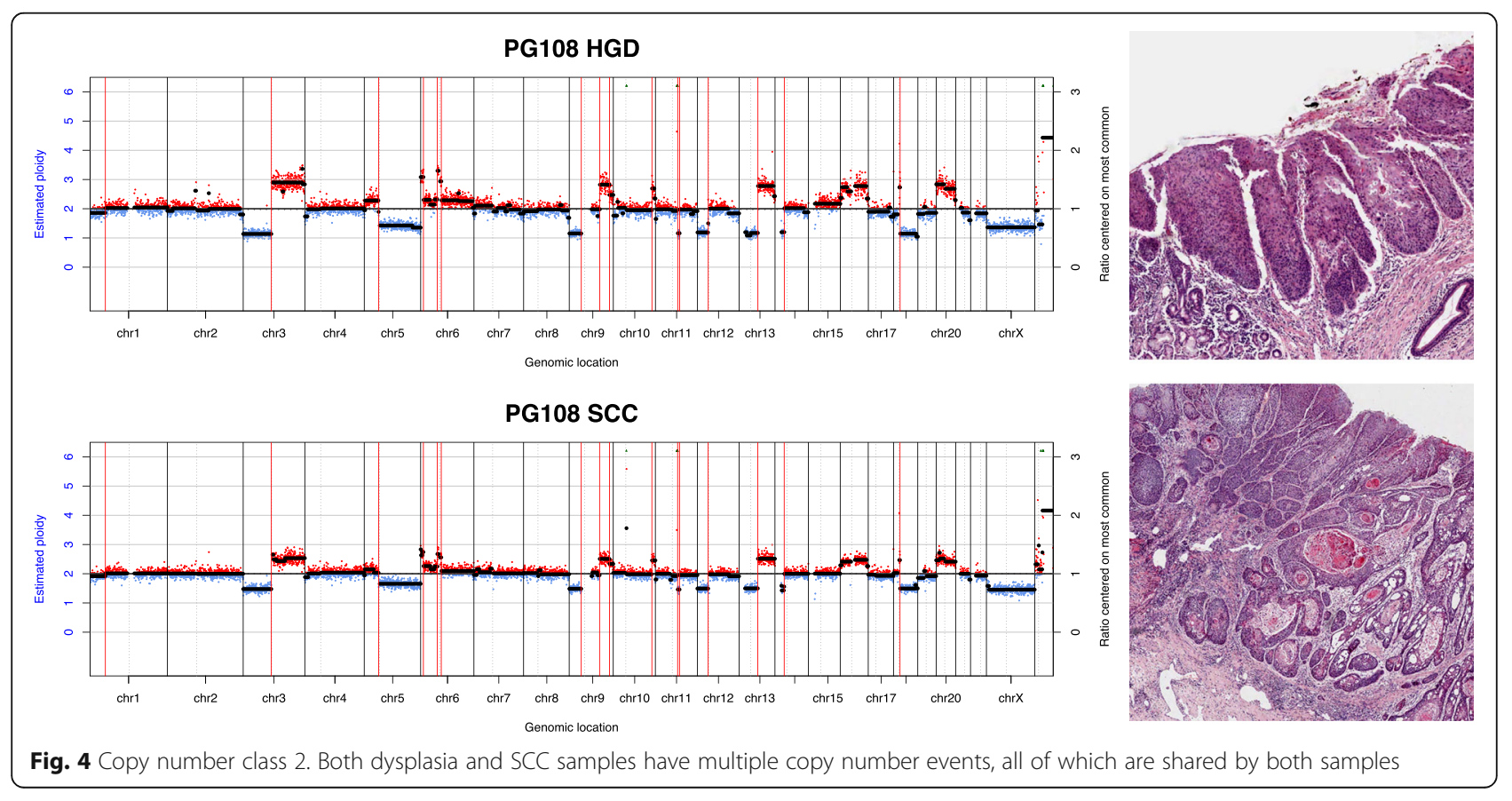




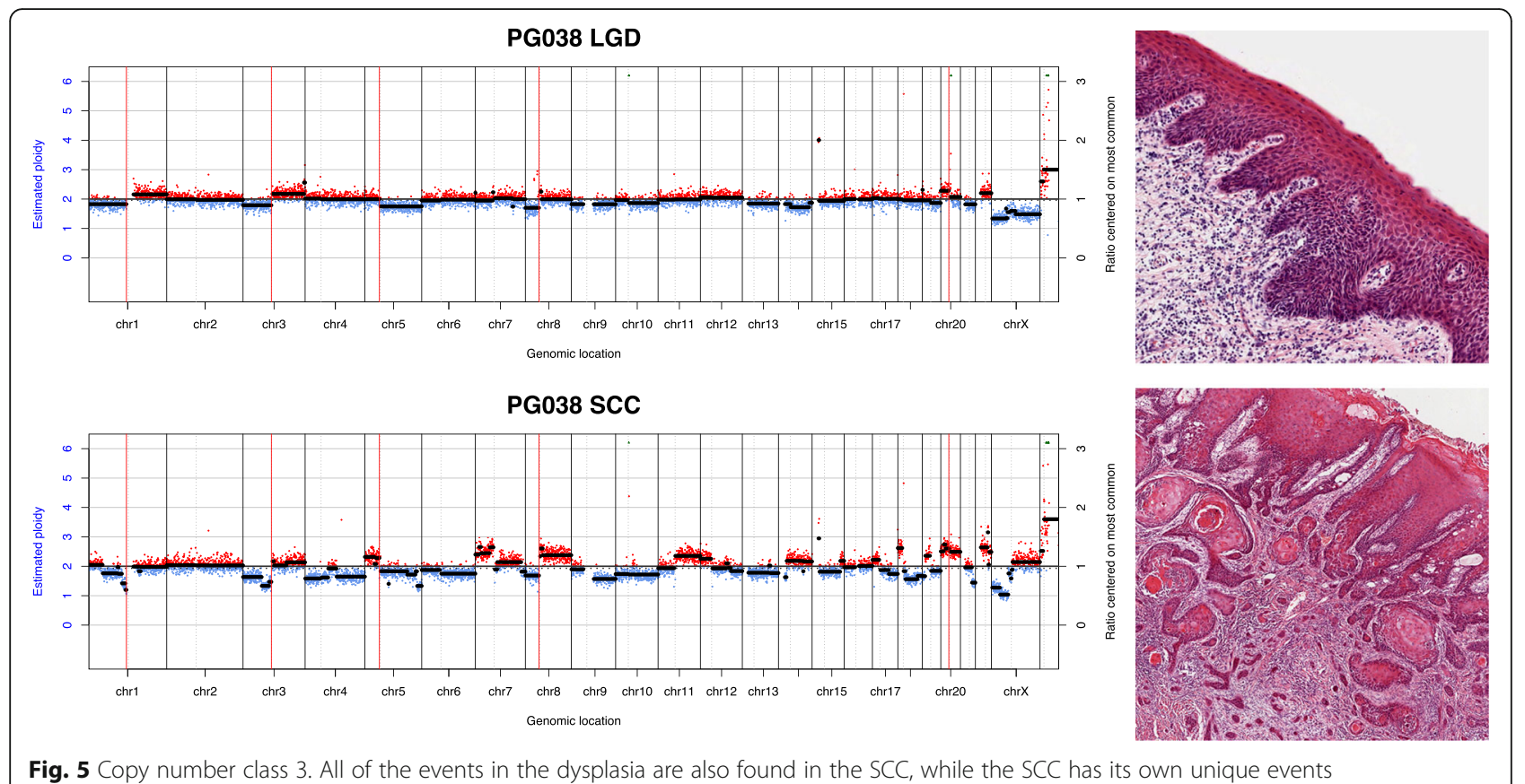

Fig. 5 Copy number class 3. All of the events in the dysplasia are also found in the SCC, while the SCC has its own unique events

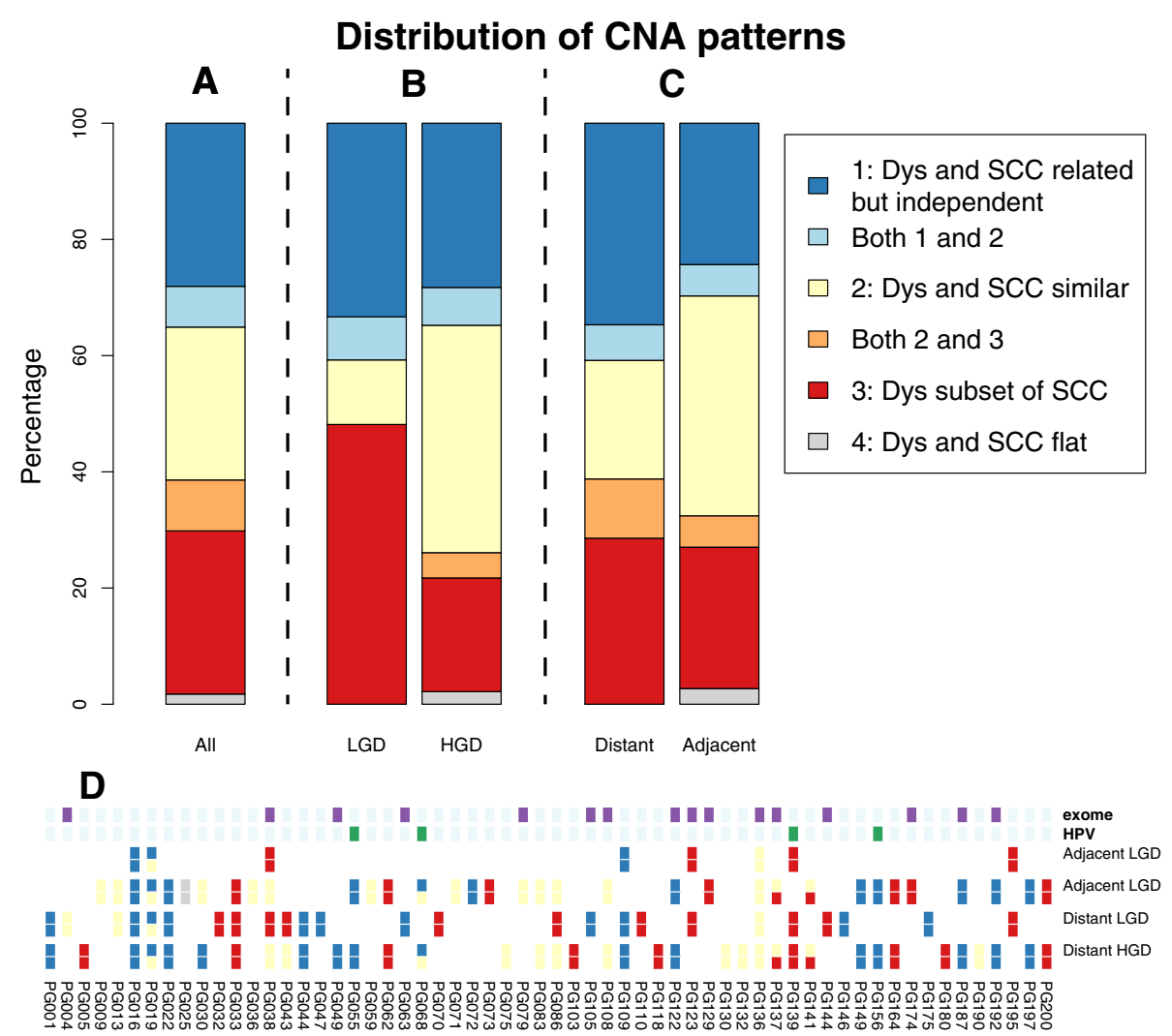

Fig. 6 Distribution of copy number alteration (CNA) patterns. a The pattern for all patients. b Comparison of LGD and HGD. c Comparison of patients with adjacent and/or distant dysplasia_SCC pairs. $\mathbf{d}$ Patterns for each individual patient, along with labels for those patients who were either HPV-positive and/or selected for exome sequencing 
lower frequency of pattern 2 and relatively higher frequencies of patterns 1 and 3 . The differences were not as striking as in the LGD-HGD comparison and, consequently, this result was not significant $(p=0.6296)$.

\section{Exome sequencing}

Sixteen patients were selected for exome sequencing of one dysplasia and one SCC sample each. Sequencing statistics for each sample are shown in Additional file 1: Table S2. Between 1146 and 7835 (median 2523) somatic variants were called in either the SCC or dysplasia per patient with initial filters in place. Relatively small numbers of these variants, between 1.9 and $15.9 \%$ (median 9.5\%) were shared between dysplasia and SCC samples from the same patient. A patient-by-patient breakdown of these results is shown in Additional file 1: Figure S3.

When VAFs from paired samples were compared, every patient had a number of private mutations and a diffuse cluster of shared mutations. An example is shown in Fig. 7, with all samples shown in Additional file 1: Figure S6. The private mutations were spread over a range of VAFs, so were not restricted to minor sub-clones. More likely they were mutations which were locally clonal but globally subclonal and simply a reflection of sampling. For all 16 patients (16 SCC and 14 dysplasias), the shared mutations had a significantly different distribution of VAFs than the total mutations, with higher values. For eight patients, this was maintained even after VAFs $<0.12$ were removed. However, the shared VAFs were not confined to the higher end of the range of values. For those samples with a significantly different distribution, the VAFs of shared events still covered the entire range, but with a shift towards higher values. Higher VAFs for shared mutations would indicate that these represented earlier, more common sub-clones, or the result of a selective sweep, with the remaining, low VAFs events being private mutations which occurred since then. The observed similar, or overlapping, distributions are more indicative of spatial sampling, with a mixture of sub-clones being spread throughout the lesion with locally variable population densities.

To ensure that these shared mutations were not simply caused by recurrent FFPE artefacts which could occur in any sample, shared mutation calls between and within patients were compared. For every sample, the number of mutation calls shared with every other sample was collected, both from the same patient and from different patients. Comparing numbers of shared calls between patients establishes a baseline level of how many shared calls are likely FFPE artefacts. These results are summarised in Additional file 1: Figure S5 and show that for every VAF range, the number of shared mutations within a patient was significantly greater than the number of presumably false calls shared between patients. For the calls with a VAF over 0.1, there was no

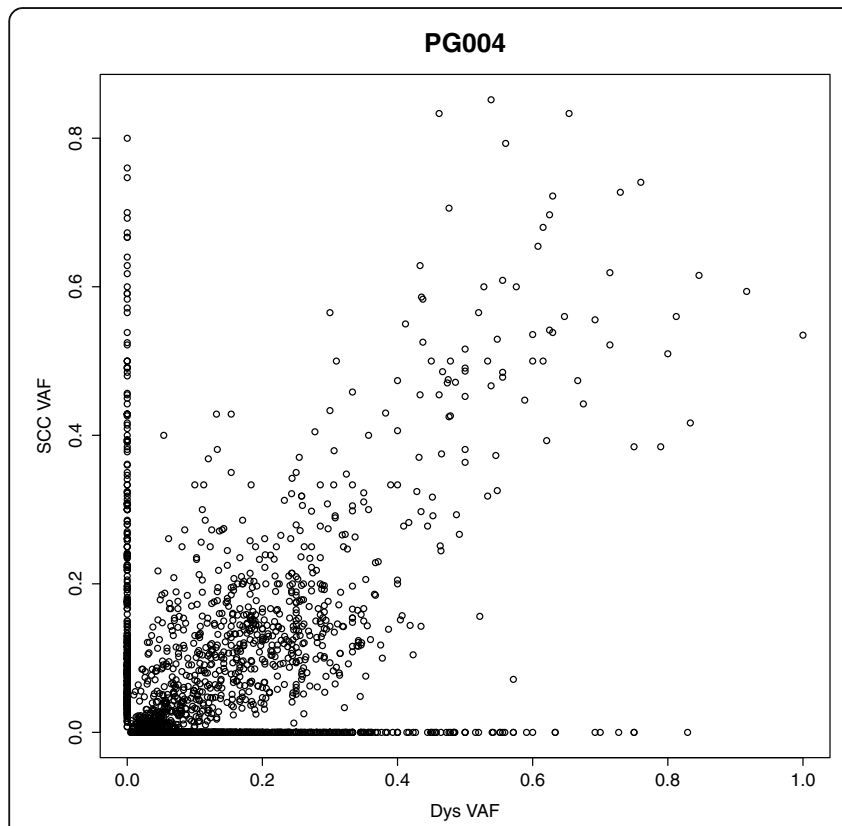

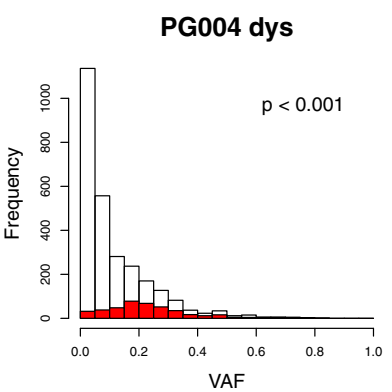

PG004 dys

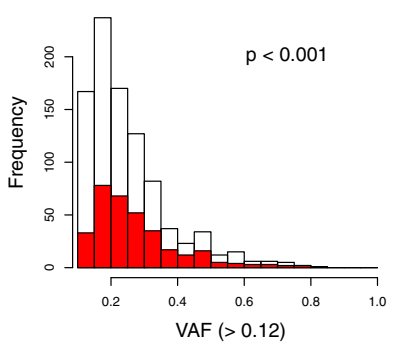

PG004 scc

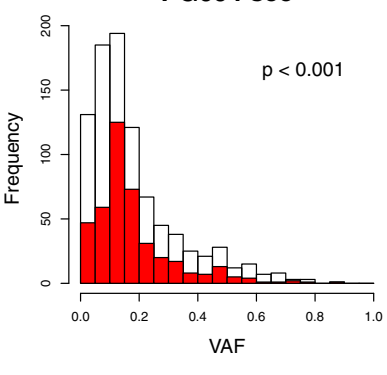

PG004 scc

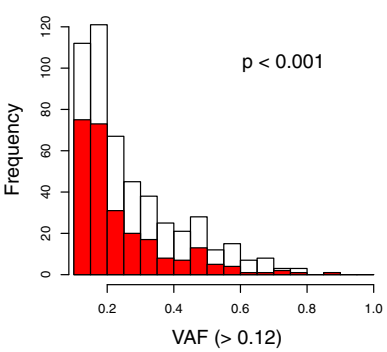

Fig. 7 Distribution of shared and private VAFs for patient PG004. In the left plot, all putative mutations are displayed as SCC VAF versus dysplasia VAF. Private mutations are on the axes at $\mathrm{x}=0$ or $\mathrm{y}=0$. To make the distributions of VAFs more visible, they are displayed as histograms in the right plots. On the left, the distribution of dysplasia VAFs is shown, with the shared mutations in red. On the right the SCC VAFs are shown. Many patients had high numbers of putative calls with very low VAFs, which may be spurious and which make the rest of the distribution difficult to see. Therefore, the bottom two plots replicate the top plots, but only with VAFs $>0.12$. For each histogram, the $p$ value showing the likelihood of the shared and total distributions being the same is shown 
overlap at all between the distributions. The sample pair with the fewest number of shared mutations within a patient had more shared calls than the pair with the most between-patient calls.

No patient showed evidence of more than one cluster of mutations, which might reflect distinct sub-clones which were rare in one sample and more common in the pair, as has been seen in leukaemia [32], and might be a result of a new, selectively advantaged sub-clone emerging.

We tested for this putative absence of sub-clonal selection by plotting the rate at which sub-clonal mutations emerged. Neutral evolution will see a steady accumulation of mutations. If a new sub-clone causes a selective advantage, then the measured number of mutations per cell division will drop, as the new sub-clone begins to dominate its surroundings, reducing heterogeneity. All 16 SCC samples and 14 of the 16 dysplasias had an $\mathrm{R}^{2}$ value over 0.98 , which has been calculated to be a stringent indicator of neutrality [6]. Examples of neutral and non-neutral samples are in Fig. 8, with all samples shown in Additional file 1: Figure S7. Both the nonneutral samples showed a reduction of the mutations per cell division over time, indicative of a selectively advantaged sub-clone emerging at an early stage. The lack of this curve in the matching SCC suggests that this clone is fixed in the population by the time the disease becomes invasive, and that further sub-clonal evolution confers no further selective advantage to growth rate. The model uses an "effective cell division rate", which is division minus cell death. It is not clear if these results show the true emergence of a sub-clone with a selective growth advantage over its neighbours, or whether the overall growth of the lesion increased as the cells moved towards a more invasive phenotype, free of the control mechanisms of normal tissue.

These results also allow for estimations of mutation rate which are less skewed by sequence errors and FFPE artefacts than mere counting of variants, using the gradient of the graphs in the region between the low VAFs, which may be artefacts, and the high VAFs, which may be clonal. While there was variation between samples (Fig. 9), all rates were in line with those taken from TCGA lung series, as calculated by Williams et al. [6]. One sample (PG192) was a clear outlier, with mutation rates more than twice that of the next highest patient. Five patients had a higher rate for dysplasia, 11 for SCC. Again, it should be noted that these rates are mutations per base per effective cell division. A putatively high rate may actually be a normal rate with low cell division-a slow growing disease.

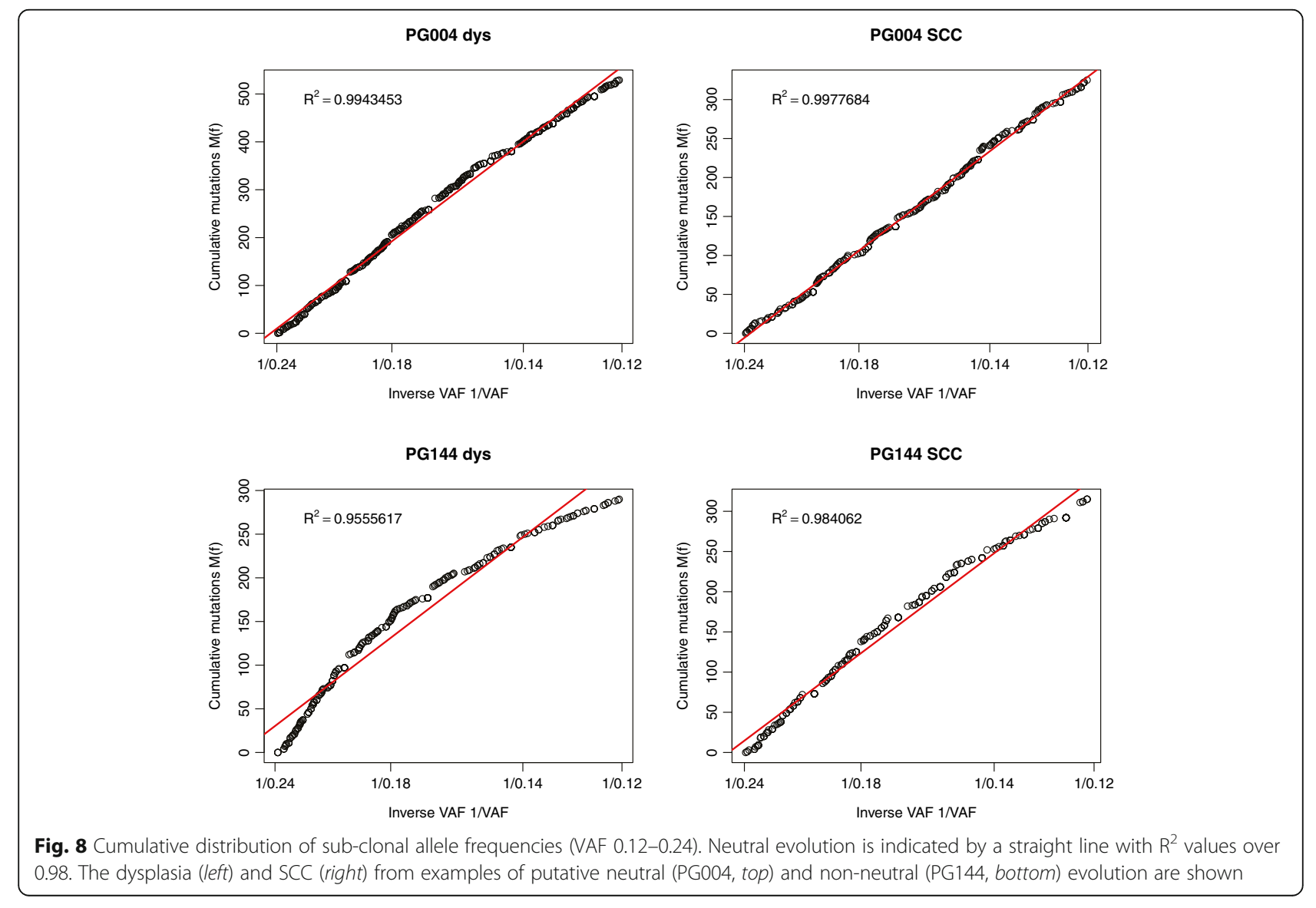




\section{Mutation Rates}

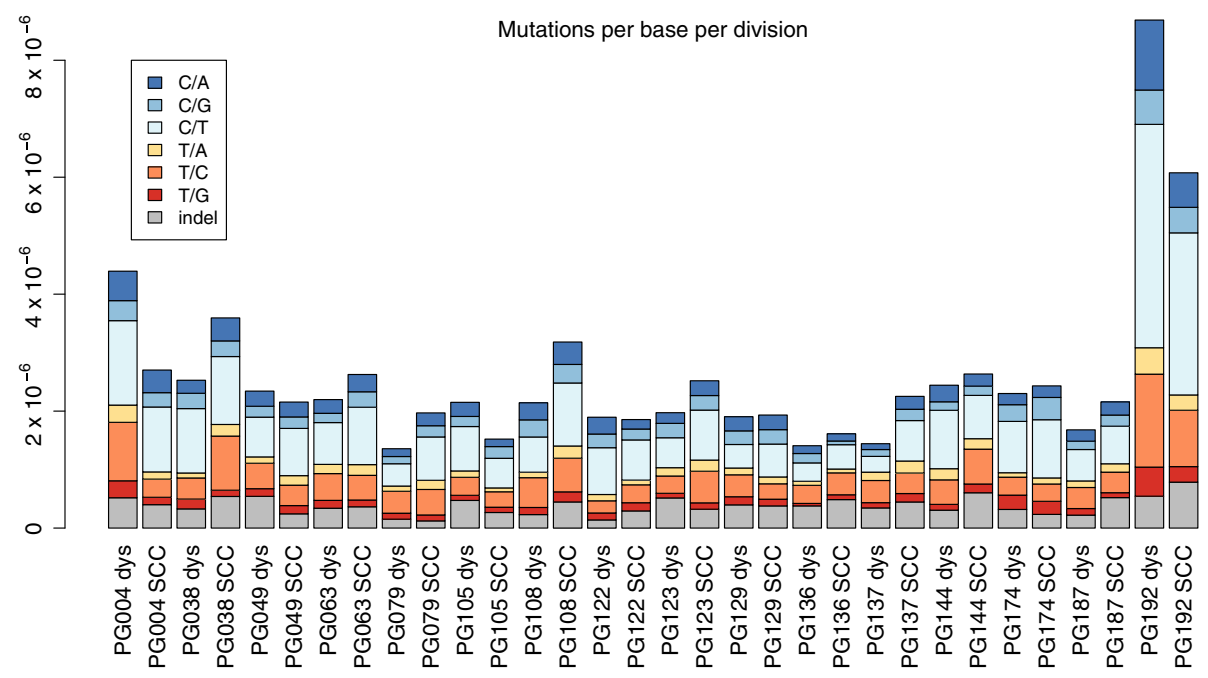

Fig. 9 Mutation rates of all samples, calculated as the gradients of the cumulative distribution plots divided by the size of the exome. Mutation types are differentiated by colour

Since we observed that most samples demonstrated essentially neutral evolution, with sub-clonal mutations having little effect on tumour growth, it appeared that potential driver events were more likely to be early, clonal mutations. Therefore, variants were filtered to remove those present in less than $50 \%$ of cells in both dysplasia and SCC samples, and those which would have no predicted effect on the protein expressed. The results of this more stringent filtering are shown in Additional file 1: Figure S4. Between 44 and 179 variants per patient passed the filters (median 97). These mutations, with their predicted effects, are listed in full in Additional file 2. The filtered variants were more likely to be shared between dysplasia and SCC samples than the unfiltered variants $(p<0.0001)$. One patient shared $2.7 \%$ variants while the rest shared between 11.8 and 53.3\% (median 34.3\%). All patients had fewer mutations in dysplasia than SCC samples. All but two had more shared mutations than dysplasia-only changes. All but one patient had more SCC-only mutations than shared. Neither the number nor proportion of shared mutations correlated with the total number of mutations per sample. Mutations are listed in Additional file 1: Table S3.

To further investigate putative driver genes, the variants were filtered against lists of genes in pathways predicted to be altered in cancer, as well as lists of genes observed to be frequently mutated in HNSCC. These results are shown in Fig. 10, Additional file 1: Figure S8 and Additional file 1: Table S3. The numbers of genes affected varied considerably between patients-between 3 and 31 (median 10). Between 0 and $67 \%$ of these were shared between samples. The relationships between the number of SCC-only, shared and dysplasia-only mutations was not as straightforward has had been seen in the numbers of filtered mutations. Only eight patients had any dysplasia-only changes in suspected cancer genes. All patients had some SCC only changes. One had no shared events. Four patients had more shared than SCC mutations, 11 had more SCC than shared, while one had equal numbers of shared and SCConly changes. No pattern could be observed linking these numbers to either dysplasia grade or whether the dysplasia was adjacent or distant to the SCC. Similarly, the proportion of shared variants was not significantly different to the initial list of filtered variants $(p=0.921)$. If these were all driver genes, then it would be expected that they would be more likely to be early, shared events than the list of filtered genes. As this was not the case, it seems that many of the genes on these list were merely potential drivers, rather than actively driving these lesions.

Particular attention was paid to genes present in over $10 \%$ of samples in TCGA HNSCC survey. TP53 was mutated in 11 of the 16 patients. For eight of these patients, the TP53 mutation was shared between the SCC and dysplasia, indicating that it was an early event. Two patients had mutated TP53 in their SCC but not their dysplasia samples (both of these dysplasia samples were in a different FFPE block to the SCC). One patient had separate TP53 mutations in both the dysplasia and the SCC, which were not shared between the two. Other TCGA genes were not shared as frequently: seven genes shared, ten SCC-only and three dysplasia-only. Interestingly, one of the samples with no TP53 mutations had the highest numbers of other common TCGA genes mutated.

Finally, the filtered variants were used to generate lists of Gene Ontology (GO) terms significantly affected by the 


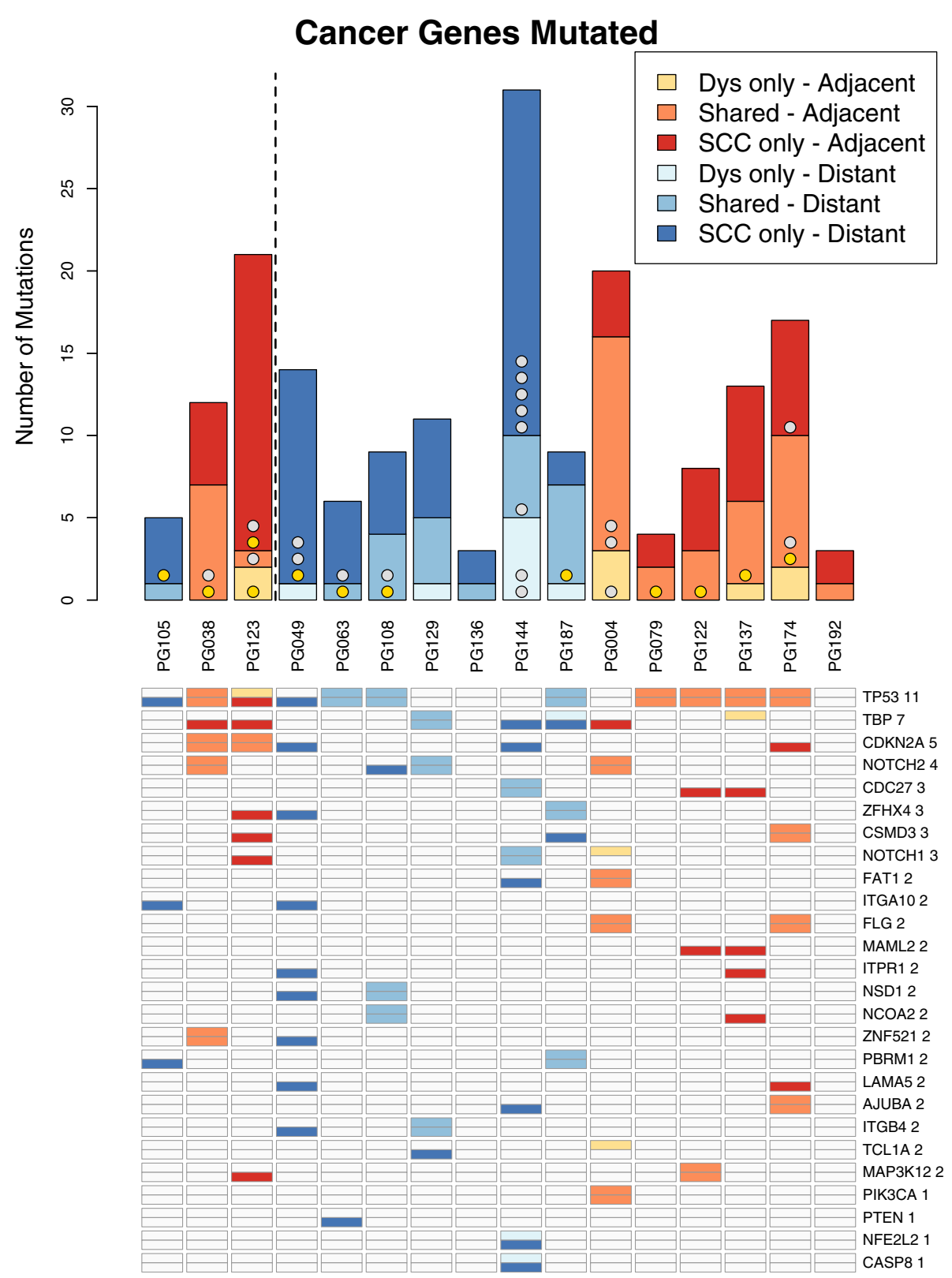

Fig. 10 The variants seen affecting putative cancer pathways or lists of common HNSCC genes. TP53 mutations are shown in gold. Other common TCGA mutations are shown in silver. Any putative cancer gene mutated in more than one patient plus all common TCGA mutations are listed. Shades of orange indicate adjacent dysplasia-SCC pairs. Shades of blue indicate distant pairs. For each patient, the number of dyplasia-only, shared and SCC-only variants are shown. There are two rows for each gene; the top row indicates that it is mutated in dysplasia and the bottom row in SCC. So for TP53, for example, PG105 has a SCC-only mutation, PG038 has a mutation which is shared, and PG123 has different mutations in the dysplasia and SCC samples. All genes are shown in Additional file 1: Figure S8

dysplasia-only, shared and SCC-only mutations from each patient. These results are shown in Additional file 1: Table S4. For 11 of the 16 patients, no dysplasia-only GO terms were significantly enriched. Every patient had some GO terms enriched that could realistically be linked to carcinogenesis, such as apoptosis, cell adhesion, cell differentiation, response to wounding, etc. However, there was little overlap between patients, or any particular pattern of some terms appearing in the shared lists and some in the SCConly lists.

\section{Discussion}

The frequent close physical association between oral dysplasia and invasive carcinoma has led to the common assumption that the relationship between the two is sequential, with one arising from the other [33-36]. A 
common approach is to observe groups of either dysplasias or carcinomas and compare them to each other, with some evidence suggesting that lower grade dysplasias have lower risk of progression [7, 8]. Recent comprehensive characterisation of fully invasive disease has suggested that either TP53 mutation or HPV infection are almost universal, along with a small number of common copy number patterns, but that changes to other genes were far more heterogeneous, with only six other genes mutated in over $10 \%$ of samples, and none of those in more than $25 \%$ [12].

The purpose of this study was to investigate the genomic similarities and differences between LGD, HGD and SCC. As well as studying groups of samples of similar grade, we were able to observe the changes in individual patients, which is important given how few common changes there are in HNSCC. Was it the case that certain groups of changes always occurred in a specific order, and how similar are the dysplasia and carcinoma samples from each patient? Additionally, this work also allowed us to test the hypothesis that dysplasia is a simpler precursor to invasive disease. Seminal work in colorectal cancer has suggested a simple step-wise genomic development from normal tissue, through pre-cancerous stages of increasing grade to fully invasive disease [2]. In previous work we have examined clonal relationships between dysplasia and carcinoma in five patients, which suggested that the relationships are sometimes complex and are not always a simple progression [15]. By working with larger numbers of patients, we were able in this study to examine trends between the different grades of disease.

By examining the frequencies of copy number gains and losses in the cohorts of LGD, HGD and SCC samples, genomic damage did appear to be linked to grade. HGD samples showed copy number patterns almost indistinguishable from those of SCC samples. Only a minority of LGD samples displayed these changes, with many changes in HGD and SCC samples absent. These findings suggest that these copy number changes were not necessary to develop LGD, and tended to occur at some point in the development from LGD to HGD. The HGD samples contained all the common copy number changes seen in the invasive disease, so the final trigger for invasion was more likely to be a gene mutation or some other transcriptomic, epigenetic or environmental change. Forty percent of dysplasia samples displayed independent evolution of the genome absent in the SCC, showing that the samples taken were not the direct progenitors of the invasive disease.

A similar pattern was observed when examining the shared and private mutations seen in the exome data. The shared mutations tended (but not overwhelmingly) to have higher VAFs, indicative of early sub-clones. The presence of large numbers of low VAF shared mutations in all samples does suggest that the same minor subclones are present in both the dysplastic and invasive part of a lesion, and that mutationally similar cells may appear histologically different, perhaps depending on the status of the majority of their neighbours. If, instead, the invasive process had begun with a single sub-clone in the dysplasia, which proceeded without mixing with any of its neighbours, then all the shared mutations would have a high VAF in the SCC sample.

We examined this gradual model of tumour evolution by testing whether mutations were accumulating according to a neutral or selective pattern. Two dysplasia samples showed evidence of early selection, but the remaining dysplasias and all SCCs followed the neutral predictions. Neutral evolution was even found in patients such as PG049, where all the known driver mutations occurred only in the SCC sample, which might suggest a late occurring selective advantage. This could be due to the fact that only one dysplasia sample was taken for each patient. More extensive sampling might have detected these mutations in a region of the dysplasia more directly ancestral to the SCC, as we sometimes found when we sampled multiple regions of a smaller group of patients [15]. An alternative explanation is that these driver mutations only affected the invasive potential of the lesions and had less effect on their growth rate. Even though we only sequenced 16 patients, no other cancer type examined by Williams et al. [6] displayed neutral evolution in more than half of the patients examined. If we were expecting $50 \%$ of samples to be neutral, observing neutrality in 14 has a $p$ value of 0.0027 . This unusually high frequency could be explained by the natural history of HNSCC. It is typically caused by decades of tobacco use, with attendant constant gradual mutation in the oral epithelium. However, in contrast with lung cancer, oral cancer is in one of the most sensitive and accessible regions of the body, so is frequently diagnosed extremely early, possibly before the emergence of later, selectively advantaged populations.

Since no samples showed evidence of a late-emerging driver clone, we concentrated on mutations in a high proportion of cells (in either dysplasia or SCC) which might have a putative effect on protein function.

As with the copy number analysis, once samples within patients were compared, the variability between patients was stark, in both numbers of overall mutations and the proportions of those mutations which were dysplasia-only, shared, or SCC-only.

Known cancer genes were very infrequently mutated only in the dysplasia samples, suggesting that whilst dysplasia may sometimes evolve away from the recent common ancestor, much of this evolution consists of the accumulation of passenger events. Cancer gene mutations were equally likely to be shared, or SCC-only, depending on the patient. TP53 mutations, however, were almost 
always shared, indicating that, where present, they were necessary, early events in the development of HNSCC.

When examining GO terms, very few terms were enriched from the dysplasia-only lists. This confirmed the hypothesis that the many mutations accumulating as dysplasia and SCC evolve away from the common ancestor are sporadic passengers. Once mutations that perturb a pathway begin to accumulate, this fraction of cells is more likely to be represented in the invasive disease. Which pathway is perturbed does not appear to be shared between patients.

\section{Conclusions}

Taken together, these results present a fascinating insight into the early stages of HNSCC development. Copy number changes and point mutations do appear to accumulate as the disease moves from normal tissue to LGD, HGD and SCC, but there does not appear to be a step-wise appearance of a wave of sub-clones, each replacing its ancestor in a selective sweep. The finding of multiple low VAF shared mutations raises the intriguing possibility that the behaviour of cells may be influenced by their neighbours, as has been observed in other cancer types [37-40]. We do see an increase in mutations and copy number changes as we move from LGD to HGD to SCC, but this does not appear to correspond to a matching change in selective advantage. It is speculation at this stage, but it could be that new sub-clones do not increase in relative frequency in a lesion because they create an environment in which their sister sub-clones can also flourish. Similar to the concept of field cancerisation [36], the long period of tobacco exposure in most patients may produce a subclinical population of cells which are primed for disease, and once a suitable combination of mutations emerges, not just the cells directly mutated change their phenotype.

Our findings could have significance for future efforts to monitor patients with dysplasia, when deciding whether or not to intervene. Although dysplasia samples did have mutations not found in the associated SCC, these were not usually in known cancer genes. Mutations in cancer genes in the dysplasia samples were mostly also found in the associated SCC samples, so could be used as markers of progression.

\section{Additional files}

Additional file 1: An example of tissue selection, a full sample list, exome sequencing metrics, full VAF and neutrality comparisons, lists of genes mutated, and lists of GO terms enriched. (PDF $8250 \mathrm{~kb}$ )

Additional file 2: For each patient undergoing exome sequencing, a full list of the mutations passing filters and their predicted effects are given in excel files. These files are the Variant Effect Predictor output for the genes present in over $50 \%$ of cells in either dysplasia or SCC, which are dysplasia-only, shared, or SCC-only. Note that due to multiple possible transcripts, most mutations are listed multiple times, with multiple possible effects. (ZIP $2331 \mathrm{~kb}$ )
Acknowledgements

Not applicable.

\section{Funding}

This work was supported by Yorkshire Cancer Research [L341PG], the Betty Woolsey Endowment, and a grant awarded to A.J. Bjourson (Ulster University) under the European Union Regional Development Fund (ERDF) EU Sustainable Competitiveness Programme for N. Ireland \& Northern Ireland Public Health Agency (HSC R\&D).

\section{Availability of data and materials}

Sequence data are available from the European Nucleotide Archive with accession number PRJEB14850 (http://www.ebi.ac.uk/ena/data/view/PRJEB14850).

\section{Authors' contributions}

HW, CC and PR designed the study. RC, LR and TO collected samples. BS, JG, NS, PC and KM provided clinical information and pathological examination. CD, RC, BS, PE and CC carried out laboratory work. HW, PC, KM, PR and CC analysed data and interpreted results. All authors read and approved the final manuscript.

\section{Competing interests}

The authors declare that they have no competing interests.

\section{Consent for publication}

Not applicable.

\section{Ethics approval and consent to participate}

All research conformed to the Declaration of Helsinki. All patients gave written informed consent. Ethical approval was granted by Leeds Teaching Hospitals. REC East Yorkshire, Leeds (East) reference numbers 07/Q1206/30 and $08 / \mathrm{H} 1306 / 127$.

\section{Publisher's Note}

Springer Nature remains neutral with regard to jurisdictional claims in published maps and institutional affiliations.

\section{Author details}

${ }^{1}$ Leeds Institute of Cancer and Pathology, University of Leeds, Leeds LS9 7TF, UK. 'St James's Institute of Oncology, St James's University Hospital, Leeds LS9 7TF, UK. ' ${ }^{3}$ eeds Dental Institute, Leeds General Infirmary, Leeds LS2 9LU, UK. ${ }^{4}$ Department of Oral Pathology, Faculty of Dentistry, Gazi University, Ankara, Turkey. ${ }^{5}$ Northern Ireland Centre for Stratified Medicine, University of Ulster, Altnagelvin Hospital, Londonderry, Northern Ireland BT47 6SB, UK.

Received: 29 March 2017 Accepted: 22 May 2017

Published online: 07 June 2017

References

1. Knudson Jr AG. Mutation and cancer: statistical study of retinoblastoma. Proc Natl Acad Sci U S A. 1971;68:820-3.

2. Vogelstein B, Fearon ER, Hamilton SR, Kern SE, Preisinger AC, Leppert M, Nakamura Y, White R, Smits AM, Bos JL. Genetic alterations during colorectal-tumor development. N Engl J Med. 1988:319:525-32.

3. Gerlinger M, Horswell S, Larkin J, Rowan AJ, Salm MP, Varela I, Fisher R, McGranahan N, Matthews N, Santos CR, et al. Genomic architecture and evolution of clear cell renal cell carcinomas defined by multiregion sequencing. Nat Genet. 2014;46:225-33.

4. Nik-Zainal S, Van Loo P, Wedge DC, Alexandrov LB, Greenman CD, Lau KW, Raine K, Jones D, Marshall J, Ramakrishna M, et al. The life history of 21 breast cancers. Cell. 2012;149:994-1007.

5. Sottoriva A, Kang H, Ma Z, Graham TA, Salomon MP, Zhao J, Marjoram P, Siegmund K, Press MF, Shibata D, Curtis C. A Big Bang model of human colorectal tumor growth. Nat Genet. 2015;47:209-16.

6. Williams MJ, Werner B, Barnes CP, Graham TA, Sottoriva A. Identification of neutral tumor evolution across cancer types. Nat Genet. 2016;48:238-44.

7. Mehanna HM, Rattay T, Smith J, McConkey CC. Treatment and follow-up of oral dysplasia - a systematic review and meta-analysis. Head Neck. 2009;31:1600-9.

8. Zhang L, Poh CF, Williams M, Laronde DM, Berean K, Gardner PJ, Jiang H, Wu L, Lee JJ, Rosin MP. Loss of heterozygosity $(\mathrm{LOH})$ profiles-validated risk predictors for progression to oral cancer. Cancer Prev Res (Phila). 2012;5:1081-9. 
9. Califano J, Westra WH, Meininger G, Corio R, Koch WM, Sidransky D. Genetic progression and clonal relationship of recurrent premalignant head and neck lesions. Clin Cancer Res. 2000;6:347-52.

10. Tsui IF, Garnis C, Poh CF. A dynamic oral cancer field: unraveling the underlying biology and its clinical implication. Am J Surg Pathol. 2009;33:1732-8.

11. Noutomi Y, Oga A, Uchida K, Okafuji M, Ita M, Kawauchi S, Furuya T, Ueyama Y, Sasaki K. Comparative genomic hybridization reveals genetic progression of oral squamous cell carcinoma from dysplasia via two different tumourigenic pathways. J Pathol. 2006;210:67-74.

12. Cancer Genome Atlas N. Comprehensive genomic characterization of head and neck squamous cell carcinomas. Nature. 2015;517:576-82.

13. Barnes L, Eveson JW, Reichart P, Sldransky D. World Health Organization classification of tumours, pathology and genetics of head and neck tumours. Lyon: IARC Press; 2005.

14. Kujan O, Oliver RJ, Khattab A, Roberts SA, Thakker N, Sloan P. Evaluation of a new binary system of grading oral epithelial dysplasia for prediction of malignant transformation. Oral Oncol. 2006:42:987-93.

15. Wood HM, Conway C, Daly C, Chalkley R, Berri S, Senguven B, Stead L, Ross $L$, Egan $P$, Chengot $P$, et al. The clonal relationships between pre-cancer and cancer revealed by ultra-deep sequencing. J Pathol. 2015;237:296-306.

16. Conway C, Graham JL, Chengot P, Daly C, Chalkley R, Ross L, Droop A, Rabbitts P, Stead LF. Elucidating drivers of oral epithelial dysplasia formation and malignant transformation to cancer using RNAseq. Oncotarget. 2015;6:40186-201

17. Wood HM, Belvedere O, Conway C, Daly C, Chalkley R, Bickerdike M, McKinley C, Egan P, Ross L, Hayward B, et al. Using next-generation sequencing for high resolution multiplex analysis of copy number variation from nanogram quantities of DNA from formalin-fixed paraffin-embedded specimens. Nucleic Acids Res. 2010;38(14):e151.

18. Martin M. Cutadapt removes adapter sequences from high-throughput sequencing reads. EMBnetjournal. 2011;17:10-12

19. Gusnanto A, Wood HM, Pawitan Y, Rabbitts P, Berri S. Correcting for cancer genome size and tumour cell content enables better estimation of copy number alterations from next-generation sequence data. Bioinformatics. 2012;28:40-7.

20. Li H, Durbin R. Fast and accurate short read alignment with BurrowsWheeler transform. Bioinformatics. 2009;25:60.

21. Venkatraman ES, Olshen AB. A faster circular binary segmentation algorithm for the analysis of array CGH data. Bioinformatics. 2007;23:657-63.

22. Conway C, Chalkley R, High A, Maclennan K, Berri S, Chengot P, Alsop M, Egan $P$, Morgan J, Taylor GR, et al. Next-generation sequencing for simultaneous determination of human papillomavirus load, subtype, and associated genomic copy number changes in tumors. J Mol Diagn. 2012;14:104-11.

23. McKenna A, Hanna M, Banks E, Sivachenko A, Cibulskis K, Kernytsky A, Garimella K, Altshuler D, Gabriel S, Daly M, DePristo MA. The Genome Analysis Toolkit: a MapReduce framework for analyzing next-generation DNA sequencing data. Genome Res. 2010;20:1297-303.

24. Koboldt DC, Zhang Q, Larson DE, Shen D, McLellan MD, Lin L, Miller CA Mardis ER, Ding L, Wilson RK. VarScan 2: somatic mutation and copy number alteration discovery in cancer by exome sequencing. Genome Res. 2012;22:568-76

25. McLaren W, Pritchard B, Rios D, Chen Y, Flicek P, Cunningham F. Deriving the consequences of genomic variants with the Ensembl API and SNP Effect Predictor. Bioinformatics. 2010;26:2069-70.

26. Kanehisa M, Goto S, Sato Y, Kawashima M, Furumichi M, Tanabe M. Data, information, knowledge and principle: back to metabolism in KEGG. Nucleic Acids Res. 2014;42:D199-205.

27. Kanehisa M, Goto S. KEGG: Kyoto Encyclopedia of Genes and Genomes. Nucleic Acids Res. 2000;28:27-30.

28. Stransky N, Egloff AM, Tward AD, Kostic AD, Cibulskis K, Sivachenko A, Kryukov GV, Lawrence MS, Sougnez C, McKenna A, et al. The mutational landscape of head and neck squamous cell carcinoma. Science. 2011;333:1157-60

29. Futreal PA, Coin L, Marshall M, Down T, Hubbard T, Wooster R, Rahman N, Stratton MR. A census of human cancer genes. Nat Rev Cancer. 2004;4:177-83.

30. da Huang W, Sherman BT, Lempicki RA. Bioinformatics enrichment tools: paths toward the comprehensive functional analysis of large gene lists. Nucleic Acids Res. 2009;37:1-13.

31. da Huang W, Sherman BT, Lempicki RA. Systematic and integrative analysis of large gene lists using DAVID bioinformatics resources. Nat Protoc. 2009;4:44-57.
32. Walter MJ, Shen D, Ding L, Shao J, Koboldt DC, Chen K, Larson DE, McLellan MD, Dooling D, Abbott R, et al. Clonal architecture of secondary acute myeloid leukemia. N Engl J Med. 2012;366:1090-8.

33. Ho MW, Risk JM, Woolgar JA, Field EA, Field JK, Steele JC, Rajlawat BP, Triantafyllou A, Rogers SN, Lowe D, Shaw RJ. The clinical determinants of malignant transformation in oral epithelial dysplasia. Oral Oncol. 2012;48:969-76.

34. Ho MW, Field EA, Field JK, Risk JM, Rajlawat BP, Rogers SN, Steele JC, Triantafyllou A, Woolgar JA, Lowe D, Shaw RJ. Outcomes of oral squamous cell carcinoma arising from oral epithelial dysplasia: rationale for monitoring premalignant oral lesions in a multidisciplinary clinic. Br J Oral Maxillofac Surg. 2013;51:594-9.

35. Brennan M, Migliorati CA, Lockhart PB, Wray D, Al-Hashimi I, Axell T, Bruce AJ, Carpenter W, Eisenberg E, Epstein JB, et al. Management of oral epithelial dysplasia: a review. Oral Surg Oral Med Oral Pathol Oral Radio Endod. 2007;103(S19):e11-2.

36. Slaughter DP, Southwick HW, Smejkal W. Field cancerization in oral stratified squamous epithelium; clinical implications of multicentric origin. Cancer. 1953:6:963-8

37. Cleary AS, Leonard TL, Gestl SA, Gunther EJ. Tumour cell heterogeneity maintained by cooperating subclones in Wnt-driven mammary cancers. Nature. 2014;508:113-7.

38. Lee Y, El Andaloussi S, Wood MJ. Exosomes and microvesicles: extracellular vesicles for genetic information transfer and gene therapy. Hum Mol Genet. 2012;21:R125-34.

39. Skog J, Wurdinger T, van Rijn S, Meijer DH, Gainche L, Sena-Esteves M, Curry Jr WT, Carter BS, Krichevsky AM, Breakefield XO. Glioblastoma microvesicles transport RNA and proteins that promote tumour growth and provide diagnostic biomarkers. Nat Cell Biol. 2008;10:1470-6.

40. Zhang J, Li S, Li L, Li M, Guo C, Yao J, Mi S. Exosome and exosomal microRNA: trafficking, sorting, and function. Genomics Proteomics Bioinformatics. 2015;13:17-24.

\section{Submit your next manuscript to BioMed Central and we will help you at every step:}

- We accept pre-submission inquiries

- Our selector tool helps you to find the most relevant journal

- We provide round the clock customer support

- Convenient online submission

- Thorough peer review

- Inclusion in PubMed and all major indexing services

- Maximum visibility for your research

Submit your manuscript at www.biomedcentral.com/submit
) Biomed Central 